\title{
An improved ARIMA model for precipitation simulations
}

\author{
H. R. Wang ${ }^{1}$, C. Wang ${ }^{1}$, X. Lin $^{2}$, and J. Kang ${ }^{2}$ \\ ${ }^{1}$ College of Water Sciences, Key Laboratory for Water and Sediment Sciences Ministry of Education, \\ Beijing Normal University, 19 Xinjiekouwai Street, Beijing, 100875, China \\ ${ }^{2}$ College of Mathematic Sciences, Beijing Normal University, 19 Xinjiekouwai Street, Beijing, 100875, China
}

Correspondence to: C. Wang (chengw@knights.ucf.edu)

Received: 20 March 2014 - Published in Nonlin. Processes Geophys. Discuss.: 30 April 2014

Revised: 6 October 2014 - Accepted: 13 October 2014 - Published: 1 December 2014

\begin{abstract}
Auto regressive integrated moving average (ARIMA) models have been widely used to calculate monthly time series data formed by interannual variations of monthly data or inter-monthly variation. However, the influence brought about by inter-monthly variations within each year is often ignored. An improved ARIMA model is developed in this study accounting for both the interannual and inter-monthly variation. In the present approach, clustering analysis is performed first to hydrologic variable time series. The characteristics of each class are then extracted and the correlation between the hydrologic variable quantity to be predicted and characteristic quantities constructed by linear regression analysis. ARIMA models are built for predicting these characteristics of each class and the hydrologic variable monthly values of year of interest are finally predicted using the modeled values of corresponding characteristics from ARIMA model and the linear regression model. A case study is conducted to predict the monthly precipitation at the Lanzhou precipitation station in Lanzhou, China, using the model, and the results show that the accuracy of the improved model is significantly higher than the seasonal model, with the mean residual achieving $9.41 \mathrm{~mm}$ and the forecast accuracy increasing by $21 \%$.
\end{abstract}

\section{Introduction}

Hydrological processes are complicated; they are influenced by not only deterministic, but also stochastic factors (Wang et al., 2007). The deterministic change in a hydrological process is always accompanied by the stochastic change. Generally speaking, determinism includes periodicity, tendency, and abrupt change. A strict deterministic hydrological process is rare. Stationary time series has been widely used in hydrological data assimilation and prediction to tackle the stochastic factors in hydrological processes. From the point of view of stochastic processes, hydrological data series usually comprises trend term and stationary term. The basic idea of the auto regressive integrated moving average (ARIMA) model, one of the most commonly used time series model, is to remove the trend term of series by difference elimination, so that a nonstationary series can be transformed into a stationary one. Some researchers have used ARIMA model for the analysis of hydrological process without considering the effects of seasonal factors (Jin et al., 1999; Niua et al., 1998; Toth and Montanari, 1999). However, most studies (Ahmad et al., 2001; Lehmann and Rode, 2001; Qi an Zhen, 2006) neglected stationary test and the influence from inter-monthly variation within a year. In this paper, the seasonal ARIMA model is improved by removing the effect of seasonal factors, and the improved model is tested through a case study. The paper is organized as follows: the ARIMA model is introduced first, followed by the introduction of the issues in the currently existing ARIMA model and our proposed methods to improve it. A case study is conducted and discussion is addressed finally.

\section{ARIMA model}

A hydrological time series $\left\{y_{t}, t=1,2, \ldots, n\right\}$ could be either stationary or nonstationary. Given that there are essentially no strictly deterministic hydrological processes in nature, the analysis of hydrological data by means of nonstationary time series is of importance, among which ARIMA model is one of the available choices. 


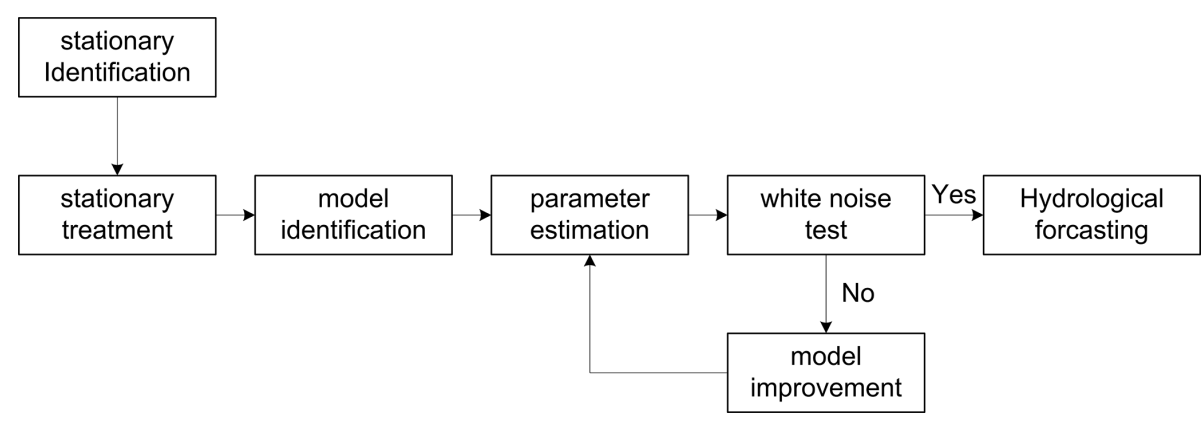

Figure 1. Procedure of applying ARIMA model.

\subsection{ARIMA model}

For a stationary time series, an auto regressive moving average model (ARMA) $(p, q)$ model is defined as follows:

$$
\begin{aligned}
y_{t} & =\phi_{1} y_{t-1}+\phi_{2} y_{t-2}+\ldots+\phi_{p} y_{t-p}+u_{t}-\theta_{1} u_{t-1} \\
& -\theta_{2} u_{t-2}-\ldots-\theta_{q} u_{t-q},
\end{aligned}
$$

where $p$ denotes the autoregressive (AR) parameters, $q$ represents the moving average (MA) parameters, the real parameters $\phi_{1}, \phi_{2}, \ldots$, and $\phi_{p}$ are called autoregressive coefficients, the real parameters $\theta_{j}(j=1,2, \ldots, q)$ are moving average coefficients, and $u_{t}$ is an independent white noise sequence, i.e., $u_{t} \sim N\left(0, \sigma^{2}\right)$. Usually the mean of $\left\{y_{t}\right\}$ is zero; if not, $y_{t}^{\prime}=y_{t}-\mu$ is used in the model.

Lag operator $(B)$ is then introduced; thus

$$
\begin{aligned}
& \varphi(B)=1-\varphi_{1} B-\varphi_{2} B^{2}-\ldots-\varphi_{p} B^{p}, \\
& \theta(B)=1-\theta_{1} B-\theta_{2} B^{2}-\ldots-\theta_{q} B^{q},
\end{aligned}
$$

where $\varphi(B)$ is the autoregressive operator and $\theta(B)$ is the moving-average operator.

Then the model can be simplified as

$\varphi(B) y_{t}=\phi(B) u_{t}$.

If $\left\{y_{t}, t=1,2, \ldots, n\right\}$ is nonstationary, we can obtain the stationarized sequence $z_{t}$ by means of difference, i.e.,

$z_{t}=(1-B)^{d} y_{t}=\nabla^{d} y_{t}$,

where $d$ is the number of regular differencing. Then the corresponding ARIMA $(p, d, q)$ model for $y_{t}$ can be built (Box, 1997), where $d$ is the number of differencing passes by which the nonstationary time series might be described as a stationary ARMA process.

\subsection{Seasonal ARIMA $(p, d, q)$ model}

Most hydrological time series have obviously seasonal (quasi-periodic) variation (Box, 1967), representing recurring of hydrological processes over a relatively (but not strictly) fixed time interval. Monthly data series often shows a seasonal period of 12 months while quarterly data series always present a period of 4 quarters. Seasonality can be determined by examining whether the autocorrelation function of the data series with a specified seasonal order is significantly different from zero. For instance, if the autocorrelation coefficient of a monthly data series with new data series formed by a lag of 12 months is not significantly different from 0 , the monthly data series does not have a seasonality of 12 months; if the autocorrelation coefficient is significantly different from 0 , it is very likely this monthly data series has a seasonality of 12 months. A seasonal ARIMA model can be built for a data series with seasonality.

For a time series $\left\{y_{t}\right\}$, its seasonality can be eliminated after $D$ orders of differencing with a period of $S$. If a further $d$ orders of regular differencing is still needed in order to make the data series stationary, a seasonal ARIMA can be built for the data series as follows,

$\phi_{p}(B) \Phi_{P}\left(B^{s}\right)(1-B)^{d}\left(1-B^{s}\right)^{D} y_{t}=\theta_{q}(B) \Theta_{Q}\left(B^{s}\right) u_{t}$

where $P$ is the number of seasonal autoregressive parameter, $Q$ is the seasonal moving average order, $s$ is the period length (in month in this work), and $D$ denotes the number of differencing passes.

\subsection{Implementation of ARIMA model}

The procedure of estimating ARIMA model is given by the flowchart in Fig. 1 which involves the following steps:

1. Stationary identification: the input time series for an ARIMA model needs to be stationary, i.e., the time series should have a constant mean, variance, and autocorrelation through time. Therefore, the stationarity of the data series needs to be identified first. If not, the non-stationary time series is then required to be stationarized. Although the stationary test, such as the unit root test and the Kwiatkowski-Phillips-Schmidt-Shin (KPSS) tests are used to identify whether a time series is stationary, plotting approaches based on scatter diagrams, autocorrelation function diagrams, and partial correlation function diagrams are also often used. The latter approach can usually not only provide information 
on the testing time series is stationary, but it can also indicate the order of the differencing which is needed to stationarize the time series. In this paper, we identify the stationarity of a time series from the autocorrelation function diagram, and partial correlation function diagram.

If a time series is identified as nonstationary, differencing is usually made to stationarize the time series. In the differencing method, the correct amount of differencing is normally the lowest order of differencing that yields a time series, which fluctuates around a well-defined mean value, and whose autocorrelation function (ACF) plot decays fairly rapidly to zero, either from above or below. The time series is often transformed for stabilizing its variance through proper transformation, e.g., logarithmic transformation. Although logarithmic transformation is commonly used to stabilize the variance of a time series rather than directly stationarize a time series, the reduction in the variance of a time series is usually helpful to reduce the order of difference in order to make it stationary.

2. Identification of the order of ARIMA model: after a time series has been stationarized, the next step is to determine the order terms of its ARIMA model, i.e., the order of differencing, $d$ for nonstationary time series, the order of auto-regression, $p$, the order of moving average, $q$, and the seasonal terms if the data series show seasonality. While one could just try some different combinations of terms and see what works best strictly, the more systematic and common way is to tentatively identify the orders of the ARIMA model by looking at the autocorrelation function (ACF) and partial autocorrelation (PACF) plots of the stationarized time series. The ACF plot is merely a bar chart of the coefficients of correlation between a time series and lags of itself, and the PACF plot presents a plot of the partial correlation coefficients between the series and lags of itself. The detailed guidelines for identifying ARIMA model parameters based on $\mathrm{ACF}$ and PACF, can be found elsewhere, e.g., Pankratz (1983) and Shumway and Stoffer (2005). It should be noted that, to be strict, the ARIMA model built in this step is actually an ARMA model with if the time series is stationary, which is in fact a special case of ARIMA model with $d=0$.

3. Estimation of ARIMA model parameters: while least square methods (linear or nonlinear) are often used for the parameter estimation, we use the maximum likelihood method (Mcleod and Sales, 1983; Melard, 1984) in this paper. A $t$ test is also performed to test the statistical significance.

4. White noise test for residual sequence: it is necessary to evaluate the established ARIMA model with estimated parameters before using it to make forecasting. We use white noise test here. If the residual sequence is not a white noise, some useful information has not been extracted and the model needs to be further tuned. The method is illustrated as follows.

Null hypothesis : $H_{0}: \operatorname{corr}\left(e_{t}, e_{t-k}\right)=0 \quad \forall k, t$

Alternative hypothesis : $H_{1}: \operatorname{corr}\left(e_{t_{0}}, e_{t_{0}-k_{0}}\right)$

$$
\exists k_{0}, t_{0}
$$

The autocorrelation of the data series is measured by the autocorrelation coefficient which is defined as

$r_{k}=\frac{\sum_{t=k+1}^{n} e_{t} e_{t-k}}{\sum_{t=1}^{n} e_{t}^{2}} \quad(k=1,2, \ldots, m)$,

where $n$ is the number of cases in a particular sample of series for the white noise test, $m$ is the maximum amount of lag. In practice, $m$ uses the value of $\left[\frac{n}{10}\right]$ when $\mathrm{n}$ is very large and $\left[\frac{n}{4}\right]$ when $n$ is small. When $n \rightarrow \infty, \sqrt{n} r_{k} \sim N(0,1)$.

The test statistics is given by

$$
Q=n(n+2) \sum_{k=1}^{m} \frac{r_{k}^{2}}{n-k} .
$$

Given the degree of confidence of $1-\alpha$, if

$Q<\chi_{\alpha}^{2}(m-p-q)$,

then $Q$ fits the $\chi^{2}$ distribution at the significance of $1-\alpha$ and the null hypothesis is accepted.

5. Hydrological forecasting: the linear least squares method is usually applied for rainfall-runoff prediction. In general, based on the $n$ observation values, the values of future $L$ time steps can be estimated (Kohn, 1986).

\section{Improvement of a conventional ARIMA model}

Seasonal ARIMA models apply for time series which arranges in order with a certain time interval or step, e.g., a month. However, in this case, while the seasonal ARIMA model is capable of dealing with the interannual variation of each monthly of a monthly data series, the information of inter-monthly variation of the time series may be lost. For example, after an order of 12 of seasonal differencing (term $S$ in a general seasonal ARIMA model) of a monthly time series, the original monthly series has been migrated to a new time series without seasonality. A nonseasonal ARIMA model is then fitted to the new time series where the inter-monthly variation of original monthly time series has also migrated to 


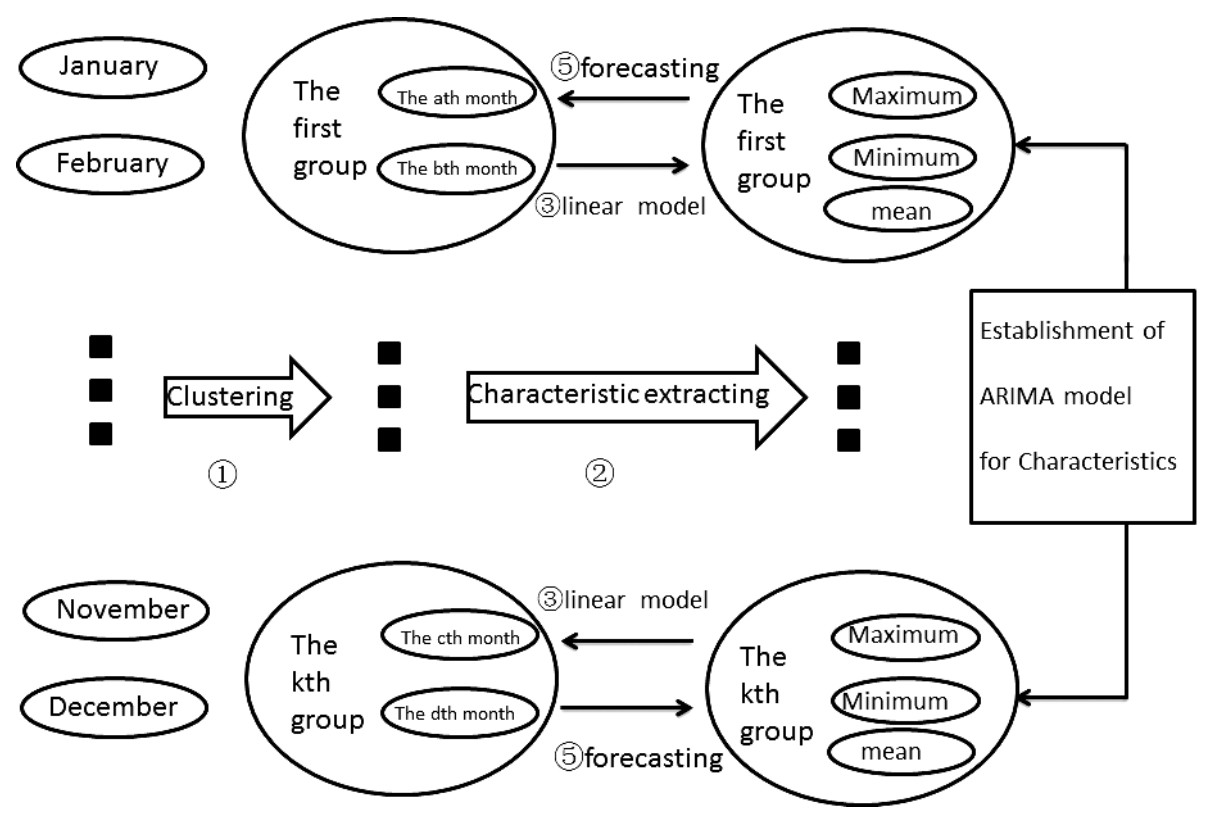

Figure 2. Prediction steps of a ARIMA model based on clustering and regressive analysis.

the inter-monthly variation of the new series after seasonal differencing. The transformation of inter-monthly variation of original monthly data to the new inter-monthly variation of seasonally differenced series may result in loss of accuracy of model performance. In this study, 12 individual seasonal ARIMA models for precipitation prediction for each month are built from each monthly data series, e.g., the January data series from 1951 to 2000, which are referred to as ARIMA models of interannual variation ignoring the inter-monthly variation.

In order to prevent from losing the inter-monthly variation information, we propose in this study the following improvement to the conventional seasonal ARIMA model, which simultaneously takes into account both kinds of temporal variation (interannual variation and inter-monthly variation). Clustering analysis is first applied to classify the monthly data series and extract characteristics of each data series class (Sun et al., 2005). In this study, we use Euclidean distance as the distance measurement in clustering analysis. The characteristics of each data series refer to the maximum, minimum, and truncated mean of the series of this class. A linear regression model is then built with hydrological variable to be predicted, e.g., monthly precipitation, as dependent variables and with maximum, minimum, and truncated mean of each class as independent variables in the linear regression model. For example, a monthly precipitation would be described as a linear regression function of the maximum, minimum, and truncated mean of the data series of a class where this month's precipitation has been clustered in the clustering analysis. A conventional seasonal ARIMA model is built for the maximum, minimum, and truncated mean of each class, respectively, accounting for the inter-monthly variation of each characteristic variable. With this method, we are trying to avoid losing the inter-monthly variation information. The implementation of the improved ARIMA model involves the following procedure, as illustrated in Fig. 2.

i. Perform clustering analysis on monthly data, and group the months with similar hydrological variation.

ii. Find the maximum, minimum, and truncated mean of each cluster.

iii. Build linear regression models and determine the associated parameters for each monthly data series. For example, for the precipitation in the $i$ th month

$y_{i}=a_{i} y_{j, \max }+b_{i} y_{j, \min }+c_{i} y_{j, \text { avg }}+d_{i}$,

where $a_{i}, b_{i}, c_{i}$, and $d_{i}$ are the coefficients in the model for the $i$ th month hydrologic parameter, e.g., precipitation, which need to be estimated, and $y_{j, \max }, y_{j, \min }$, and $y_{j, \text { avg }}$ are respectively the maximum, minimum, and truncated mean of the $j$ th class where the time series of the $i$ th month is identified in cluster analysis.

iv. Build ARIMA models for the maximum, minimum, and truncated mean of each class and predict the characteristics for the time year of interest using the established ARIMA models.

v. Substitute the predicted characteristics into the linear regression model built in Eq. (10) and obtain the monthly hydrologic variable, say precipitation. 

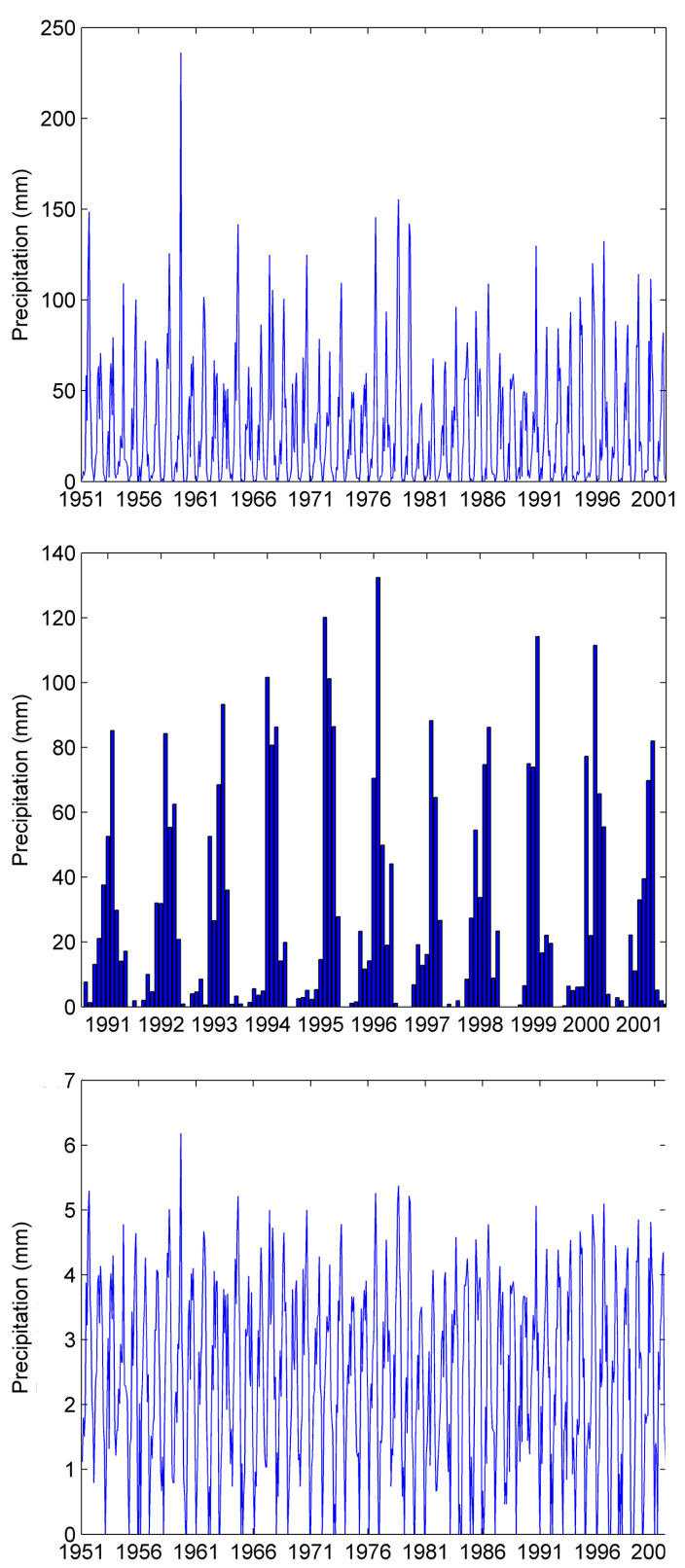

Figure 3. Monthly precipitation at the Lanzhou precipitation station. Upper panel: observation (1951-2001); middle panel: observation (1991-2000); lower panel: after power transformation (1951-2001).

\section{Case study}

In this section, we are presenting an application of the proposed improved ARIMA model to the precipitation forecasting of the Lanzhou precipitation station in Lanzhou, China. Lanzhou is located in the upper basin of Yellow River. It has a continental climate of mid-temperate zone, with an average precipitation of $360 \mathrm{~mm}$ and mean temperature of $10^{\circ} \mathrm{C}$. In general, rainfall seasons are May through September, while drought occurs in spring and winter. The Lanzhou
Table 1. Estimated parameters of the conventional seasonal ARMA model.

\begin{tabular}{lrrrc}
\hline Parameter & $\begin{array}{r}\text { Estimated } \\
\text { value }\end{array}$ & $\begin{array}{r}\text { Standard } \\
\text { deviation }\end{array}$ & $t$ test & $\begin{array}{c}\text { Tail } \\
\text { probability }\end{array}$ \\
\hline$\theta_{1}$ & -0.16379 & 0.03959 & -4.14 & $<.0001$ \\
$\theta_{2}$ & 0.93434 & 0.02117 & 44.14 & $<.0001$ \\
\hline
\end{tabular}

precipitation station is located at $103.70^{\circ} \mathrm{E}, 35.90^{\circ} \mathrm{N}$. The monthly precipitation data from 1951 to 2000 is used for parameter estimation and the monthly precipitations of 2001 are then predicted using the proposed model and compared with the observation values. In order to show the improvement of this present approach, we first build a conventional seasonal ARIMA model and a set of 12 ARIMA models for each monthly precipitation series which account for the seasonal variation. The improved ARIMA model accounting for both inter-month and interannual variation of monthly precipitation time series is then built using the presented approach and its prediction results are compared with the conventional ARIMA model and seasonal ARIMA model, as well as auto-regressive models.

\subsection{Conventional seasonal ARMA modeling}

The precipitation at the Lanzhou precipitation station from 1951 through 2001 and from 1991 through 2001 are plotted as shown in Fig. 3a and b, respectively. The two figures show less precipitation in winter and spring and more in summer and autumn. Fluctuation in the data occurs during high precipitation seasons. Using power transformation with an order of $1 / 3$, fluctuations at high values are removed and the data become stationary, as shown in Fig. 3c. According to autocorrelation and partial correlation functions, as shown in Fig. 4, seasonal term with a period of 12 exists. With the difference elimination method, the order of the model can be determined, and the following seasonal ARIMA model is obtained.

$\left(1-B^{12}\right) y_{t}=\left(1-\theta_{1} B\right)\left(1-\theta_{2} B^{12}\right) u_{t}$

The maximum-likelihood method is then used for parameter estimation and the results are listed in Table 1. As shown in Table 1, parameter estimation is statistically significant. A white noise test is performed for the residual sequence. If the test does not pass, the model needs to be improved. As shown in Table 2, with a significance level of $5 \%$, the test is passed, i.e., the useful information is extracted and the model is acceptable.

\subsection{Individual ARIMA model for each month's data series}

As discussed in Sect. 2.2, the data can be classified into 12 groups associated with each month, respectively. 
Table 2. Autocorrelation of the residuals of the conventional seasonal ARIMA model.

\begin{tabular}{lrrcrrrrrr}
\hline $\begin{array}{l}\text { AR } \\
\text { order }\end{array}$ & $\begin{array}{r}\chi^{2} \\
\text { statistic }\end{array}$ & $\begin{array}{r}\text { Degree of } \\
\text { freedom }\end{array}$ & $\begin{array}{c}\text { Tail } \\
\text { probability }\end{array}$ \\
\hline 6 & 0.770 & 4 & 0.943 & 0.000 & -0.007 & -0.018 & 0.021 & -0.007 & 0.020 \\
12 & 6.910 & 10 & 0.734 & 0.013 & 0.014 & 0.012 & -0.043 & 0.086 & -0.019 \\
18 & 13.400 & 16 & 0.643 & 0.092 & 0.014 & 0.031 & -0.004 & 0.021 & 0.020 \\
24 & 16.810 & 22 & 0.774 & 0.042 & 0.007 & -0.022 & -0.026 & -0.032 & 0.039 \\
30 & 20.650 & 28 & 0.840 & 0.050 & -0.031 & -0.048 & 0.003 & 0.018 & 0.008 \\
36 & 28.100 & 34 & 0.752 & 0.045 & 0.018 & 0.064 & -0.044 & 0.036 & 0.044 \\
42 & 30.900 & 40 & 0.849 & 0.057 & -0.015 & 0.019 & 0.023 & 0.006 & -0.001 \\
48 & 52.940 & 46 & 0.224 & -0.012 & 0.040 & -0.022 & 0.032 & -0.079 & -0.156 \\
\hline
\end{tabular}

* Autocorrelations of residue for lag 1 through lag 48, 6 lags per row from column 5 through 10 .
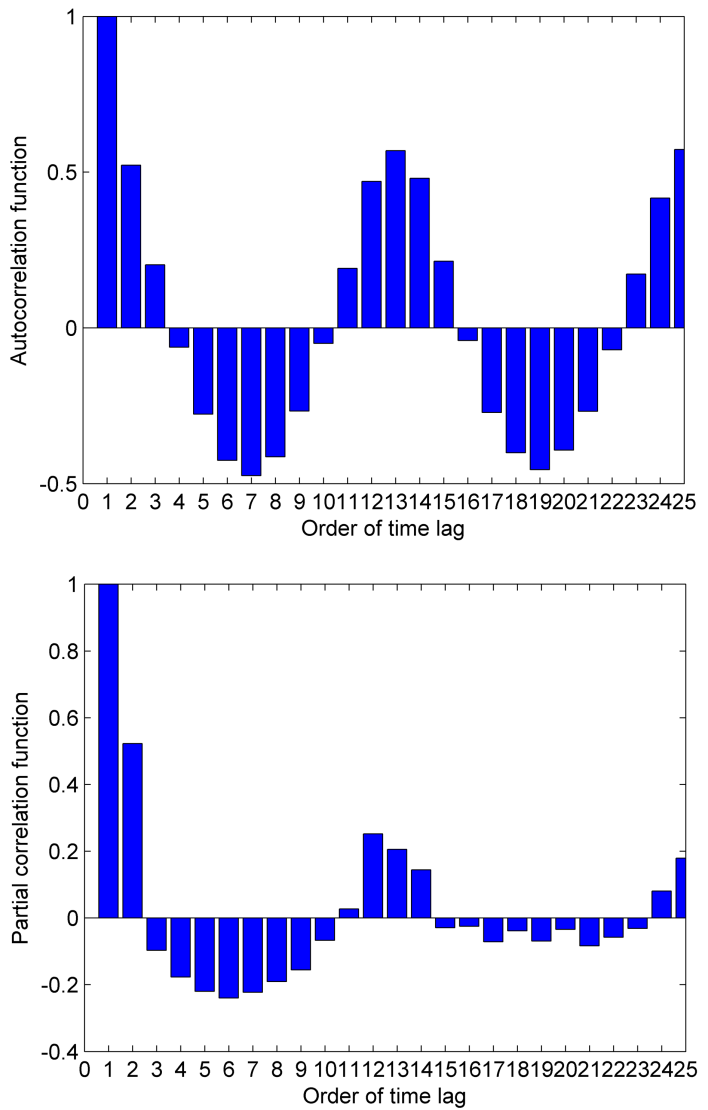

Figure 4. Autocorrelation and partial correlation plots of data series. Upper panel: autocorrelation; lower panel: partial correlation.

Stationary identification, stationary treatment, model identification, parameter estimation and residual test are performed for the 12 groups of data. The ACF and PACF plots after seasonal differencing are presented in Fig. 5. A total of 12 ARIMA models are built and the estimated parameters are shown in Table 3.
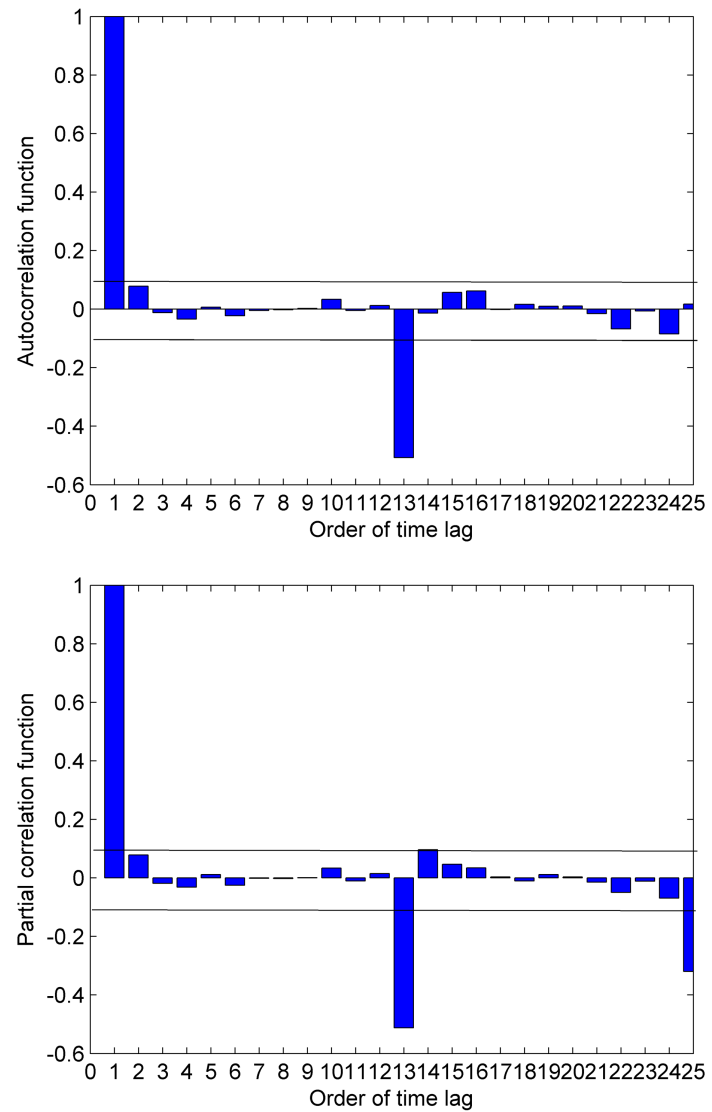

Figure 5. Autocorrelation and partial correlation plots of data series after differencing. Upper panel: autocorrelation; lower panel: partial correlation.

\subsection{The improved ARIMA model based on clustering and regression analysis}

The Box-Cox transformation is applied as a pretreatment of data for clustering analysis in order to stabilize the variance of the monthly precipitation data series. Given that the precipitation has values of zero resulting in negative infinity in the transformation, the Box-Cox transformation (Thyer et 

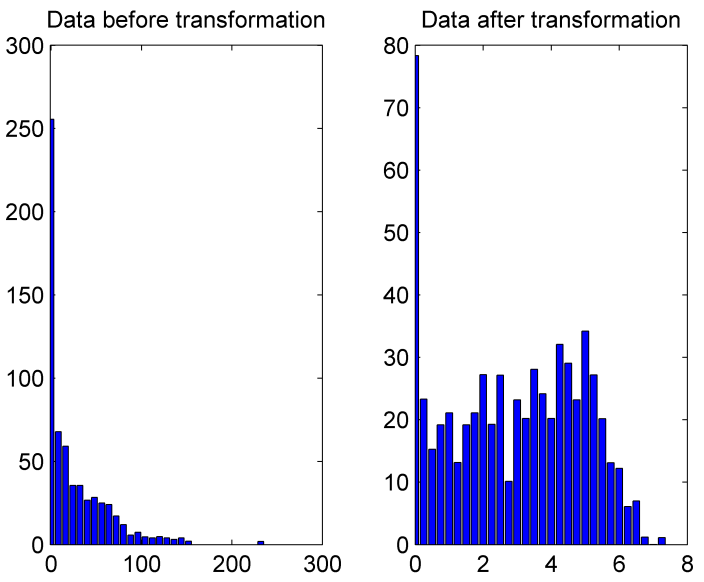

Figure 6. Monthly precipitation series before and after the Box-Cox transformation.

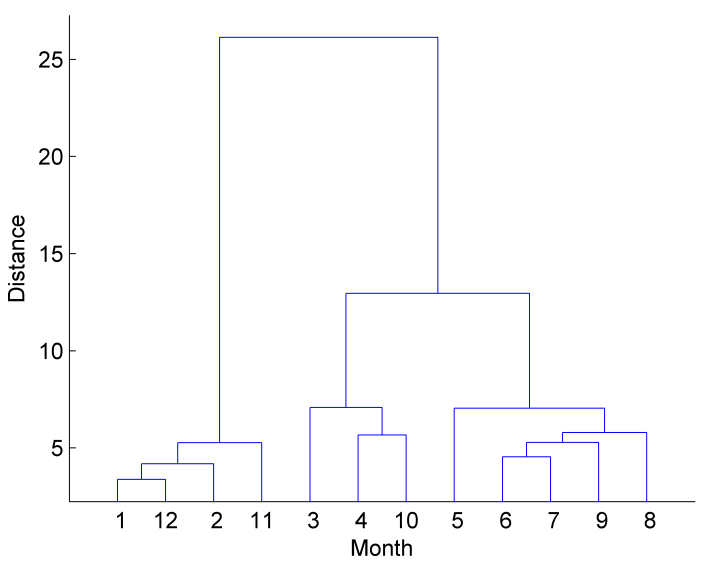

Figure 7. Clusters of monthly precipitation time series.

al., 2002; Meloun et al., 2005; Ip et al., 2004) is corrected as follows.

Data after transformation $= \begin{cases}\frac{(\text { original data }+1)^{\alpha}-1}{\alpha} & \alpha \neq 0 \\ \log (\text { original data }) & \alpha=0\end{cases}$

After the Box-Cox transformation, as shown in Fig. 6, the data are much more symmetric than the original data series, which is helpful for the later clustering analysis. Moreover, it can be seen that there are many zero precipitation values in the raw monthly precipitation data series and so does the transferred data. This indicates that the samples of data sequence may not be from one individual population but from multiple populations which further implies the necessarily of clustering analysis for the data series. Clustering analysis with Euclidean distance is then applied which indicates that the monthly precipitation sequences can be clustered into three classes, as shown in Fig. 7.
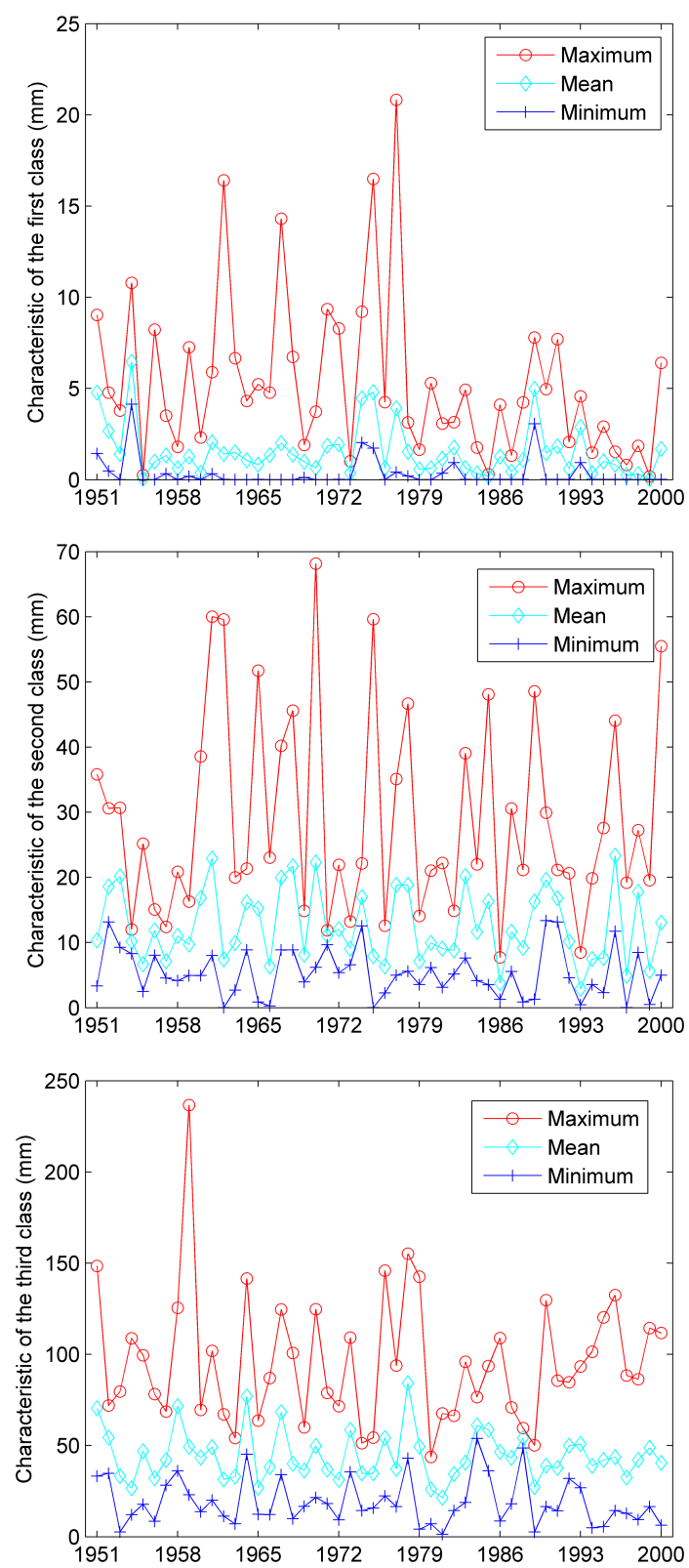

Figure 8. Characteristics of each time series class. Upper panel: first class; middle panel: second class; lower panel: third class.

Class 1: Jan., Feb., Nov., and Dec.

Class 2: Mar., Apr., and Oct.

Class 3: May, Jun., Jul., Aug., and Sep.

It is interesting that the clustering results are mostly coincides with the precipitation season. For example, Class 1 looks like it corresponds to the drought season while Class 3 corresponds to the rainfall season. After the clustering analysis to the monthly precipitation time series, the characteristics of each class, i.e., maximum, minimum, and truncated mean, are identified, as shown in Fig. 8. Although fluctuations in the mean and minimum data series are relatively 
Table 3. Seasonal ARIMA models for each month.

\begin{tabular}{lll}
\hline Month & Model & ML parameter estimation \\
\hline 1 & $(1-\alpha B) y_{t}=(1-\beta B) u_{t}$ & $\alpha=-0.95, \beta=-0.97$ \\
2 & $\left(1-\alpha B^{2}\right) y_{t}=u_{t}$ & $\alpha=-0.49$ \\
3 & $y_{t}=(1-\beta B) u_{t}$ & $\beta=0.38$ \\
4 & $y_{t}=\left(1-\beta_{1} B-\beta_{2} B^{2}\right) u_{t}$ & $\beta_{1}=0.27, \beta_{2}=-0.22$ \\
5 & $y_{t}=\left(1-\beta B^{2}\right) u_{t}$ & $\beta=-0.30$ \\
6 & $y_{t}=(1-\beta B) u_{t}$ & $\beta=-0.32$ \\
7 & $y_{t}=\left(1-\beta B^{2}\right) u_{t}$ & $\beta=-0.3349$ \\
8 & $(1-\alpha B) y_{t}=(1-\beta B) u_{t}$ & $\alpha=-0.182, \beta=-0.0528$ \\
9 & $(1-\alpha B) y_{t}=(1-\beta B) u_{t}$ & $\alpha=0.956, \beta=0.469$ \\
10 & $y_{t}=(1-\beta B) u_{t}$ & $\beta=-0.32$ \\
11 & $(1-\alpha B) y_{t}=(1-\beta B) u_{t}$ & $\alpha=0.681, \beta=0.741$ \\
12 & $(1-\alpha B) y_{t}=(1-\beta B) u_{t}$ & $\alpha=0.650, \beta=0.766$ \\
\hline
\end{tabular}

small, relatively larger variation are shown in the maximum data series.

Linear regression models for each monthly precipitation are fitted using the characteristics of each class where the monthly precipitation data series is located. The parameters corresponding to each linear regression model are presented in Table 4 which pass the $t$ test at the significance of 0.05 indicating that those linear models fit their data series well respectively. Following the steps described in Sect. 2.3, nine ARIMA modes are built for each of the characteristic variables of each class. The estimated parameters are shown in Table 5. Auto-regressive models with orders of 24 and 36, or $\mathrm{AR}(24)$ and $\mathrm{AR}(36)$, are also fitted to the monthly precipitation time series for comparative study with the improved ARIMA model and conventional ARIMA model.

\section{Results and discussion}

The monthly precipitations of 2001 are predicted using the improved ARIMA model as well as the conventional seasonal ARIMA model, the 12 seasonal ARIMA models for the precipitation of each month, and $\mathrm{AR}(24)$ and $\mathrm{AR}(36)$ models, the prediction results shown in Table 6 and Fig. 9. The absolute error of each method is 9.41, 11.49, 11.78, 17.05, and $17.82 \mathrm{~mm}$ for the improved ARIMA model, conventional ARIMA model, individual ARIMA for each month's data series, $\mathrm{AR}(24)$, and $\mathrm{AR}(36)$, respectively, indicating that the improved ARIMA presented in this paper performs the best with the smallest errors. Compared with the conventional ARIMA model, the improved ARIMA model increases the prediction accuracy by $24 \%$.

The conventional ARIMA model predicts accurately for March, June, August, and November but mismatches the other months' precipitation. It predicts more accurately for October precipitation than the improved ARIMA model. The 12 individual ARIMA models for each month data series performs similarly to the conventional ARIMA model. The overall performance of $\mathrm{AR}(24)$ model does not show
Table 4. Estimated parameters for linear regression models.

\begin{tabular}{lrrrcr}
\hline Class & Month & $d_{i}^{*}$ & $a_{i}^{*}$ & $c_{i}^{*}$ & $b_{i}^{*}$ \\
\hline \multirow{3}{*}{1} & 1 & 0.16 & 0.09 & 0.39 & 0.23 \\
& 2 & 0.21 & -0.12 & 1.21 & -0.14 \\
& 11 & -0.54 & 0.30 & 1.51 & -0.62 \\
& 12 & 0.16 & -0.27 & 0.89 & 0.53 \\
\hline & 3 & 1.92 & -0.50 & 0.46 & 0.53 \\
& 4 & -0.39 & -0.57 & 2.33 & -0.62 \\
& 10 & -1.53 & 1.07 & 0.21 & 0.09 \\
\hline & 5 & 2.17 & -0.41 & 0.22 & 0.98 \\
3 & 6 & -0.19 & -0.22 & 1.49 & -0.35 \\
& 7 & -0.22 & 0.27 & 1.05 & -0.35 \\
& 8 & -2.11 & 1.07 & 0.24 & 0.05 \\
& 9 & 0.35 & -0.72 & 2.01 & -0.33 \\
\hline
\end{tabular}

* See Eq. (10) for definition.

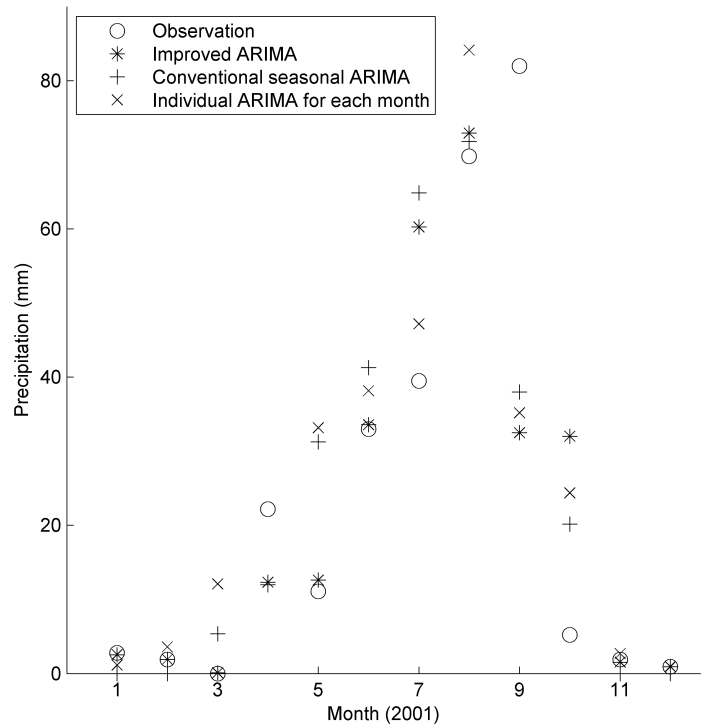

Figure 9. Comparison between predictions and observation.

difference from that of $\mathrm{AR}(36)$ model; neither models perform as good as the improved ARIMA model or the conventional ARIMA model. However, the AR models give a better prediction for September precipitation of 2001 than the other two models.

While the improved ARIMA model catches the correct trend overall and predicts the monthly precipitation in most months with high accuracy, it predicts highly accurately for the dry seasons, such as January, February, March, November, and December. However, it overestimates the precipitation of July and October and underestimates the September precipitation significantly. After a closer look at the data, we find that the mean precipitations of July and October are 63.8 and $23.48 \mathrm{~mm}$ over the period of 1951 through 2000 , respectively, whereas the observation precipitations of both 
Table 5. Parameters of ARIMA models for characteristic variables of each class.

\begin{tabular}{|c|c|c|c|c|c|c|}
\hline Class & $\begin{array}{l}\text { Characteristic } \\
\text { variable }\end{array}$ & ARIMA model & $\begin{array}{l}\text { ML parameter } \\
\text { estimating }\end{array}$ & & $\begin{array}{c}\text { Standard deviation } \\
\text { estimating }\end{array}$ & Value of $P$ \\
\hline \multirow{3}{*}{1} & maximum & $(1-B)(1-\alpha B) y_{t}=u_{t}$ & \multicolumn{2}{|l|}{-0.56} & 0.13 & $<0.0001$ \\
\hline & mean & $(1-B) y_{t}=(1-\beta B) u_{t}$ & \multicolumn{2}{|l|}{0.92} & 0.07 & $<0.0001$ \\
\hline & minimum & $(1-B)^{2} y_{t}=(1-\beta B)^{2} u_{t}$ & \multicolumn{2}{|l|}{0.84} & 0.09 & $<0.0001$ \\
\hline \multirow{3}{*}{2} & maximum & $(1-B) y_{t}=(1-\beta B)^{2} u_{t}$ & \multicolumn{2}{|l|}{-0.30} & 0.14 & 0.00311 \\
\hline & mean & $\left(1-\alpha B^{2}\right)(1-B)^{2} y_{t}=u_{t}$ & \multicolumn{2}{|l|}{-0.52} & 0.12 & $<0.0001$ \\
\hline & minimum & $\left(1-\alpha B^{2}\right)(1-B)^{2} y_{t}=u_{t}$ & \multicolumn{2}{|l|}{-0.64} & 0.11 & $<0.001$ \\
\hline \multirow{3}{*}{3} & maximum & $\left(1-\alpha B^{2}\right)(1-B)^{2} y_{t}=u_{t}$ & -0.45 & & 0.13 & 0.0006 \\
\hline & mean & $(1-\alpha B)^{2}(1-B)^{2} y_{t}=\left(1-\beta B^{4}\right) u_{t}$ & $-0.82 \quad 0.81$ & 0.20 & $<0.0001$ & \\
\hline & minimum & $(1-\alpha B)^{2}(1-B)^{2} y_{t}=\left(1-\beta B^{4}\right) u_{t}$ & -0.81 & 0.12 & $<0.0001$ & \\
\hline
\end{tabular}

Table 6. Predicted monthly precipitation data for 2001 .

\begin{tabular}{|c|c|c|c|c|c|c|c|c|c|c|c|}
\hline \multirow[t]{2}{*}{$\begin{array}{l}\text { Month } \\
(2001)\end{array}$} & \multirow[t]{2}{*}{$\begin{array}{r}\text { Observation } \\
(\mathrm{mm})\end{array}$} & \multicolumn{2}{|c|}{$\begin{array}{c}\text { Prediction by } \\
\text { improved ARIMA } \\
\text { model }(\mathrm{mm})\end{array}$} & \multicolumn{2}{|c|}{$\begin{array}{c}\text { Prediction by } \\
\text { conventional ARMA } \\
\text { model }(\mathrm{mm})\end{array}$} & \multicolumn{2}{|c|}{$\begin{array}{c}\text { Prediction by } 12 \\
\text { seasonal ARIMA } \\
\text { models }(\mathrm{mm})\end{array}$} & \multicolumn{2}{|c|}{$\begin{array}{l}\text { Prediction by AR(24) } \\
\text { model }(\mathrm{mm})\end{array}$} & \multicolumn{2}{|c|}{$\begin{array}{c}\text { Prediction by } \mathrm{AR}(36) \\
\text { model }(\mathrm{mm})\end{array}$} \\
\hline & & prediction & residual & prediction & residual & prediction & residual & prediction & residual & prediction & residual \\
\hline 1 & 2.8 & 2.54 & -0.25 & 0 & -2.8 & 1.14 & -1.66 & 0.27 & -2.53 & 0.57 & -2.23 \\
\hline 2 & 1.9 & 1.897 & -0.003 & 0 & -1.9 & 3.58 & 1.68 & 6.4 & 4.5 & 6.4 & 4.5 \\
\hline 3 & 0 & 0.099 & 0.099 & 5.38 & 5.38 & 12.10 & 12.10 & 4.89 & 4.89 & 5.24 & 5.24 \\
\hline 4 & 22.2 & 12.32 & -9.871 & 11.99 & -10.21 & 12.32 & -9.88 & 5.81 & -16.3 & 7.25 & -14.9 \\
\hline 5 & 11.1 & 12.61 & 1.515 & 31.26 & 20.16 & 33.17 & 22.07 & 6.49 & -4.61 & 12.05 & 0.95 \\
\hline 6 & 33 & 33.58 & 0.582 & 41.28 & 8.28 & 38.16 & 5.16 & 77.86 & 44.86 & 79.75 & 46.75 \\
\hline 7 & 39.5 & 60.26 & 20.76 & 64.88 & 25.38 & 47.19 & 7.69 & 22.55 & -16.9 & 20.09 & -19.4 \\
\hline 8 & 69.8 & 72.92 & 3.12 & 71.82 & 2.02 & 84.12 & 14.32 & 110.5 & 40.72 & 114.5 & 44.73 \\
\hline 9 & 82 & 32.5 & -49.5 & 37.98 & -44.02 & 35.17 & -46.83 & 65.89 & -16.11 & 63.2 & -18.8 \\
\hline 10 & 5.2 & 32.03 & 26.83 & 20.15 & 14.95 & 24.37 & 19.17 & 55.45 & 50.25 & 58.78 & 53.58 \\
\hline 11 & 1.9 & 1.532 & -0.368 & 0 & -1.9 & 2.68 & 0.78 & 3.9 & 2 & 3.79 & 1.89 \\
\hline 12 & 0.9 & 0.898 & -0.002 & 0 & -0.9 & 0.94 & 0.04 & 0 & -0.9 & 0 & -0.9 \\
\hline \multicolumn{2}{|c|}{ Mean absolute error (mm) } & \multicolumn{2}{|c|}{9.41} & \multicolumn{2}{|c|}{11.49} & \multicolumn{2}{|c|}{11.78} & \multicolumn{2}{|c|}{17.05} & \multicolumn{2}{|c|}{17.82} \\
\hline
\end{tabular}

months in 2001 are 39.5 and $5.2 \mathrm{~mm}$, respectively, much lower than the average precipitation of those 2 months. Over the 51-year period of 1951-2001, precipitation levels in July and October, 2001 are the 8th and 14th smallest, respectively. However, precipitation levels in July and October, 2001 are the 2nd and 3rd smallest from 1991 to 2001, respectively, and significantly smaller than the precipitation of other months. This may be the reason that the improved and conventional model produces underestimates for these 2 months. However, it is interesting that the AR models underestimate the July precipitation but overestimates the October precipitation. This may be because of the much lower precipitation in July, 2000 and much higher precipitation in October, 2000, relative to the July and October in 2001, which, we believe, dominate the prediction of AR models. Similarly, the September precipitation of 2000 is close to the precipitation of September in 2001, which results a better AR prediction in that month. According to the performance of AR models, we expect an improvement if we apply AR model to stationarized data series rather than the raw data series.
While the mean precipitation of September is $44.99 \mathrm{~mm}$ over the period of 1951 through 2000, the observation of September in 2001 is $82 \mathrm{~mm}$, the 4th largest one from 1951-2001, and the largest on in past 45 years. Furthermore, September 2001 is the only month in which precipitation is greater than precipitation in August throughout the previous 10 years. These facts clearly show that the precipitation level of September 2001 is an extreme value, or outlier, from a statistical point of view. Therefore, it is fair to conclude that the built ARIMA model needs to be further improved for extreme situations.

Given that both the interannual variation and intermonthly variation of the hydrological data effect the prediction of hydrological time series, it is better to account for both in order to ensure better prediction. Inter-monthly data may result from different populations as well as nonstationary factors, so the conventional seasonal ARIMA models which usually neglect the inter-monthly variations is not effective enough. An improved ARIMA model has been built in this paper taking account for both interannual and inter-monthly variation of hydrological data. Based on clustering analysis 
and regression, much more information is extracted from the data series. A case study is conducted for the precipitation at the Lanzhou precipitation station with the improved ARIMA model and the comparison with the conventional ARIMA model indicates that the accuracy of the improved ARIMA model is significantly higher than that of the conventional ARIMA model. This improved approach can be applicable to other hydrological processes prediction with time series data, such as runoff, water level, and water temperature.

Apparently, the present model could be further improved, especially for the prediction of extreme phenomena. Given that the selection of clustering method does affect model performance, different clustering methods, e.g., the definition of distance in the hierarchical clustering can be applied (Wang et al., 2005) to obtain better fittings. Characteristics value should be constructed by the features of hydrological time series, not limited to the extreme or mean values. A higher order of regression model rather than the linear regression may be used for the hydrologic forecasting. Last but not the least, artificial intelligence approaches, such as neural network or support vector machine, can be used to further improve the proposed ARIMA model.

Acknowledgements. This work was supported by the National Hi-Tech Research and Development Program of China ("863" Project, Grant No. 2012BAB02B04) and the National Natural Science Foundation of China (Grant No. 51279006). The authors are grateful to R. V. Donner and F. Yusof, whose comments greatly improved the quality of the initial manuscript.

Edited by: D. Maraun

Reviewed by: R. V. Donner and F. Yusof

\section{References}

Ahmad, S., Khan, I. H., and Parida, B. P.: Performance of stochastic approaches for forecasting river water quality, Water Res., 35, 4261-4266, 2001.

Box, G. E. P.: Models for forecasting seasonal and nonseasonal time series, in: Spectral Analysis of Time Series, edited by: Harris, B., Wiley, New York, 271-311, 1967.

Box, G. E. P.: Time Series Analysis Forecasting And Control, China Statistics Press, Beijing, China, 1997.
Ip, W. C., Wong, H., and Wang, S. G.: A GIC rule for assessing data transformation in regression, Statist. Probab. Lett., 68, 105-110, 2004.

Jin, J. L., Ding, J., and Wei, Y. M.: Threshold autoregressive model based on genetic algorithm and its application to forecasting the shallow groundwater level, Hydraul. Eng., 27, 51-55, 1999.

Kohn, R.: Estimation, prediction, and interpolation for ARIMA models with missing data, J. Am. Stat. Assoc., 81, 751-761, 1986.

Lehmann, A. and Rode, M.: Long-term behaviour and crosscorrelation water quality analysis of the River Elbe, Germany, Water Res., 35, 2153-2160, 2001.

Mcleod, A. I. and Sales, P. R. H.: An algorithm for approximate likelihood calculation of ARMA and seasonal ARMA models, Appl. Stat., 32, 211-223, 1983.

Melard, G.: A fast algorithm for the exact likelihood of autoregressive-moving average models, Appl. Stat., 33, 104-119, 1984.

Meloun, M., Sáňka, M., and Němec, P.: The analysis of soil cores polluted with certain metals using the Box-Cox transformation, Environ. Pollut., 137, 273-280, 2005.

Niua, X. F., Edmiston, H. L., and Bailey, G. O.: Time series models for salinity and other environmental factors in the Apalachicola estuarine system. Estuarine, Coast. Shelf Sci., 46, 549-563, 1998.

Pankratz, A.: Forecasting with Univariate Box-Jenkins Models: Concepts and Cases, Wiley, New York, 1983.

Qi, W. and Zhen, M. P.: Winters and ARIMA model analysis of the lake level of salt Lake Zabuye, Tibetan Plateau, J. Lake Sci., 18, 21-28, 2006.

Shumway, R. H. and Stoffer, D. S.: Time Series Analysis and Its Applications Springer-Verlag New York, Inc., Secaucus, NJ, USA, 2005.

Sun, L. H., Zhao, Z. G., and Xu, L.: Study of summer rain pattern in monsoon region of east China and its circulation cause, J. Appl. Meteorol. Sci., 16, 57-62, 2005.

Thyer, M., Kuczera, G., and Wang, Q. J.: Quantifying parameter uncertainty in stochastic models using the Box-Cox transformation, J. Hydrol., 265, 246-257, 2002.

Toth, E. A. and Montanari, B. A.: Real-time flood forecasting via combined use of conceptual and stochastic models, Phys. Chem. Earth B, 24, 793-798, 1999.

Wang, H. R., Ye, L. T., and Liu, C. M.: Problems in wavelet analysis of hydrologic series and some suggestions on improvement, Prog. Nat. Sci., 17, 80-86, 2007.

Wang, Y., Yin, L. Z., and Zhang, Y.: Judging model of fuzzy optimal dividing based on improved objective function clustering method, Math. Pract. Theory, 35, 142-147, 2005. 